\title{
Aspectos espacial e temporal e fatores associados à interiorização da Covid-19 em Pernambuco, Brasil
}

\author{
Spatial and temporal aspects and factors associated with the spread \\ of Covid-19 to the interior of the State of Pernambuco, Brazil
}

Louisiana Regadas de Macedo Quinino (https://orcid.org/0000-0002-7123-8089) ${ }^{1}$

Flávia Helena Manhães Vasconcellos (https://orcid.org/0000-0002-3053-1924) ${ }^{2}$

Isabel Soares Diniz (https://orcid.org/0000-0002-3255-3967) ${ }^{3}$

Lucilene Rafael Aguiar (https://orcid.org/0000-0003-4286-0378) 4,5

Yasmim Talita de Moraes Ramos (https://orcid.org/0000-0003-0394-8473) ${ }^{1}$

Fernanda De Bastiani (https://orcid.org/0000-0001-8532-639X) ${ }^{3}$

\footnotetext{
${ }^{1}$ Núcleo de Saúde Coletiva, Instituto Aggeu Magalhães, Fundação Oswaldo

Cruz (Fiocruz). Av. Prof.

Moraes Rego s/n, Cidade

Universitária. 50670-420

Recife PE Brasil.

louisiana_quinino@

hotmail.com

${ }^{2}$ Departamento de

Engenharia Cartográfica,

Universidade Federal de

Pernambuco (UFPE). Recife

PE Brasil.

${ }^{3}$ Departamento de Estatística, UFPE. Recife PE

Brasil.

${ }^{4}$ Faculdade de Enfermagem

Nossa Senhora das

Graças, Universidade de

Pernambuco. Recife PE

Brasil.

${ }^{5}$ Secretaria Executiva de global Moran test was applied. The first cases were recorded in and around Recife. The spread of the virus took place in neighboring municipalities and in those served by major highways. The highest incidence in MRR (640.22/100,000 inhabitants) was correlated to larger population and demographic density and lower rate of urbanization and IDH-M. Inland, the incidence (361.29/100,000 inhabitants) was correlated with a larger population, demographic density and urbanization rate, and a smaller area $(p<0.05)$. The areas of greatest risk were concentrated in MRR, but the interior showed areas of transition, indicating a tendency to spread $(p<0.05)$. Being aware of the spatial distribution pattern of Covid-19, combined with the associated factors, may contribute to greater effectiveness of control actions.
}

Vigilância Epidemiológica,

Secretaria Estadual de Saúde

de Pernambuco. Recife PE

Brasil.

\begin{abstract}
This study involved an ecological study between March and June 2020 consisting of a spatial and temporal analysis of the spread of Covid-19 to the interior of Pernambuco and related socioeconomic factors. Maps were generated from the incidence coefficient by municipality of residence to determine the spatial dynamics of Covid-19. The correlation between socioeconom$i c$ indicators and the coefficient in the metropolitan regions of Recife (MRR) and inland regions was studied. This coefficient was levelled, and the
\end{abstract}

Key words Covid-19, Spatial analysis, Epidemiology, Risk
Resumo Análise espacial e temporal da interiorização da Covid-19 em Pernambuco, considerando-se os fatores socioeconômicos correlacionados. Estudo ecológico, março a junho de 2020. Geraram-se mapas a partir do coeficiente de incidência por município de residência para determinar a dinâmica espacial da Covid-19. Estudou-se a correlação entre indicadores socioeconômicos $e$ o coeficiente nas regiões metropolitana do Recife $(R M R)$ e interior. Suavizou-se este coeficiente e calculou-se o teste de Moran global. Os primeiros casos foram registrados em Recife e arredores. $O$ caminho para a autoctonia se deu em municípios vizinhos e naqueles supridos por grandes rodovias. A maior incidência na RMR $(640,22 / 100.000$ hab) correlacionou-se à maior população e densidade demográfica e menor taxa de urbanização e IDH-M. No interior a incidência (361,29/100.000 hab) esteve correlacionada à maior população, densidade demográfica e taxa de urbanização, e menor área $(p<0,05)$. As áreas de maior risco concentraram-se na RMR, mas o interior apresentou áreas de transição, indicando tendência à interiorização $(p<0,05)$. Ter ciência do padrão de distribuição espacial da Covid-19, aliada aos fatores associados poderão contribuir para maior efetividade das ações de controle.

Palavras-chave Covid-19, Análise espacial, Epidemiologia, Risco 


\section{Introdução}

Doença restrita à China até meados de dezembro de 2019, a Covid-19 ganhou visibilidade mundial quando em 30 de janeiro de 2020 foi declarada como Emergência em Saúde de Importância Internacional (ESPII) pela Organização Mundial de Saúde (OMS). Em março de 2020, o vírus já afetava mais de 118.000 pessoas e causava mais de 4.000 mortes em 114 países, incluindo o Brasil. A partir de então, medidas precisaram ser adotadas em nível individual e coletivo, exigindo capacidade de compreensão e adaptação das pessoas e dos serviços de saúde às exigências sanitárias internacionais ${ }^{1,2}$.

Em vários países, seguir tais recomendações esbarrou na necessidade de enfrentamento de iniquidades sociais ${ }^{3,4}$. Pesquisas mostram que onde o acesso aos serviços de saúde é desigual, e onde imperam iniquidades socioeconômicas, $o$ impacto de uma pandemia recai de forma mais problemática e a adesão às recomendações fica comprometida ${ }^{3,4}$. Estudos revelam maior prejuízo para áreas rurais, dadas as vulnerabilidades relacionadas ao menor acesso à informação e a profissionais e serviços de saúde, além de terem maior contingente de população idosa ${ }^{5-8}$.

O Brasil teve seu primeiro caso confirmado de Covid-19 em 26 de fevereiro de 2020, e o segundo confirmado três dias após, ambos importados. Em 5 de março foi confirmado o primeiro caso Autóctone ${ }^{9}$. Em meio a este cenário, o país, que já convive com a necessidade de enfrentar tripla carga de doenças, teve que incluir o enfrentamento da Covid-19 em seu hall de obrigações. Atrela-se a isso a acentuada polarização política com discordância entre os governos Federal, estaduais e municipais quanto às normas propostas para contenção da pandemia, o que pode ter retardado a adoção de medidas necessárias ${ }^{3}$.

Estes fatores se agravam ainda mais quando se considera a atual característica de expansão da Covid-19 para áreas rurais, sendo o corolário deste fato o aumento da demanda por serviços de saúde em geral, principalmente leitos hospitalares gerais e de UTI. Usar o conhecimento epidemiológico para entender este fenômeno, abordando as diferenças socioeconômicas entre estas duas populações e usando ferramentas de estatística e geoprocessamento, é primordial para o enfrentamento oportuno do problema pelo sistema de saúde, refletindo na saúde das coletividades ${ }^{7-10}$.

Pernambuco foi o terceiro estado do Nordeste brasileiro a ter casos de Covid- $19^{11}$ Estudos mostram que existe heterogeneidade na forma de apresentação da Covid-19 entre e dentro dos estados do Nordeste, e que Pernambuco vem se destacando negativamente no número de óbitos. Nas primeiras semanas epidemiológicas, a doença estava restrita à região metropolitana que, destarte as iniquidades sociais, concentram as melhores infraestruturas, serviços de saúde e educação e tem as maiores ofertas de empregos, se alastrando, posteriormente, para o interior ${ }^{9-11}$.

Até onde se pôde pesquisar, os estudos publicados sobre Covid-19 em Pernambuco não abordam a interiorização da doença. Neste sentido, e considerando a importância deste assunto para a saúde pública, este trabalho visa estudar a evolução espacial e temporal da incidência da Covid-19 em Pernambuco, observando os fatores associados à interiorização da mesma.

\section{Método}

Estudo ecológico conduzido nos 184 municípios de Pernambuco a partir dos casos de Covid-19 confirmados entre 12 de março (primeiro registro em Pernambuco) e 30 de junho de 2020 por município de residência. $\mathrm{O}$ distrito de Fernando de Noronha foi excluído por não fazer fronteira com os demais ${ }^{12,13}$.

Pernambuco, situado no Nordeste do Brasil, é dividido em 184 municípios mais o distrito de Fernando de Noronha. Possui extensão territorial de $98.146,315 \mathrm{~km}^{2}$ com população estimada de 9.345.173 habitantes para o ano de 2019, sendo $80 \%$ residentes em área urbana. Apresenta Índice de Desenvolvimento Humano (IDH) de 0,673, Índice de Gini de 0,567 e Produto Interno Bruto (PIB) per capita em torno de $\mathrm{R} \$ 19,3$ mil $^{12-14}$.

A Região Metropolitana do Recife (RMR) é formada por 15 municípios (Figura 1): Abreu e Lima, Araçoiaba, Cabo de Santo Agostinho, Camaragibe, Goiana, Igarassu, Ilha de Itamaracá, Ipojuca, Itapissuma, Jaboatão dos Guararapes, Moreno, Paulista, Recife (mais populosa) e São Lourenço da Mata; possui uma população de aproximadamente 4 milhões de habitantes e uma densidade demográfica de 1,3 mil hab $/ \mathrm{km}^{2}$. Embora componha um dos quinze grandes espaços urbanos metropolitanos brasileiros (Geubs), detendo o segundo maior PIB dentre os localizados nas regiões Norte e Nordeste (36.124 milhões), apresenta desigualdades sociais ${ }^{12-14}$.

O raciocínio epidemiológico empregado neste estudo para entender a dinâmica da transmissão local da Covid-19 em Pernambuco buscou identificar e caracterizar descritivamente os 


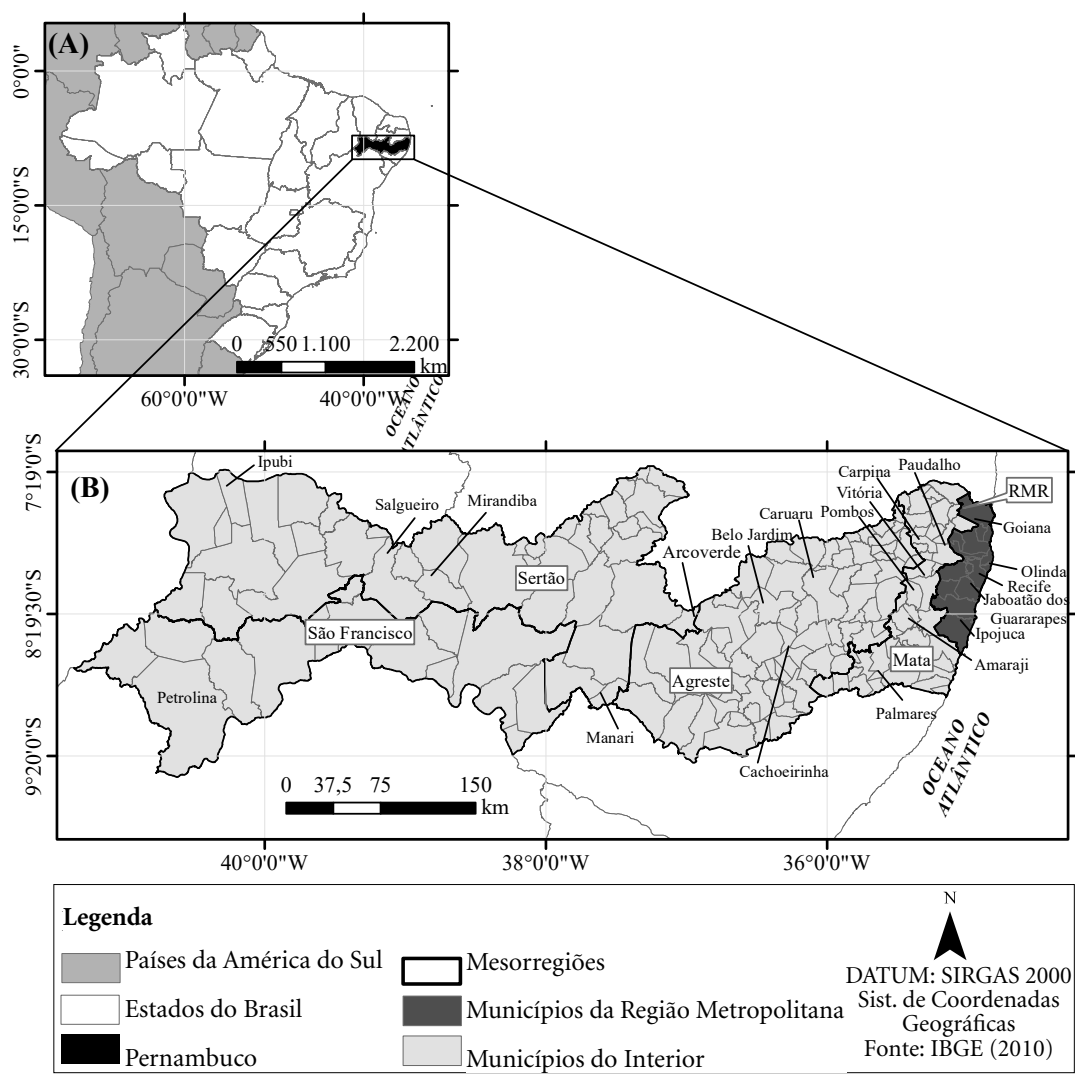

Figura 1. Localização da área de estudo: (A) Mapa do Brasil destacando a região nordeste; (B) Região nordeste destacando o estado de Pernambuco; (C) Divisão do estado de Pernambuco em mesorregiões.

Fonte: autoria própria.

primeiros municípios do estado a apresentarem casos alóctones até chegar a autoctonia, assim como mostrar a evolução espacial e temporal da Covid-19. Assim, para o plano de análise, inicialmente, mapas de distribuição dos casos foram elaborados através do software ArcGIS v.10.3.1, utilizando a malha de municípios do sítio eletrônico do IBGE (https://www.ibge.gov.br/) ${ }^{14}$ e os casos confirmados de Covid-19 (https://covid. saude.gov.br $)^{15}$. Os cortes temporais foram semanais entre as Semanas Epidemiológicas (SE) 12 e 17 e quinzenais da 18 a 27, utilizando-se o intervalo geométrico para representar os dados ${ }^{16}$. Visando verificar a interferência das grandes rodovias na transmissão da Covid-19, inseriram-se duas camadas vetoriais das BR-232 e BR-101.

Em seguida, foram apresentados, para estes municípios (Recife, Jaboatão dos Guararapes, Goiana e Olinda, na RMR, e Belo Jardim, Caru- aru, Petrolina e Palmares, no interior) e para a RMR e o interior, o coeficiente de incidência (por 100.000 habitantes), a data de surgimento dos primeiros casos, a área geográfica, a população, a densidade populacional, o IDH-M (média geométrica dos índices das dimensões renda, educação e longevidade, com mesmo peso), o Índice de Gini, o total de estabelecimentos econômicos de toda natureza e a taxa de urbanização.

Informações sobre número de casos foram coletados no site oficial (https://covid.saude.gov. $\mathrm{br} /)^{15}$. Dados populacionais (população exposta ao risco de adoecer por Covid-19 no período) foram coletados do site do Instituto Brasileiro de Geografia e Estatística (IBGE) (https://www. ibge.gov.br/ $/)^{14}$. Informações sobre território e ambiente, demográficas, sociais e econômicas foram coletadas do IBGE e do site Base de Dados do Estado (BDE) (http://www.bde.pe.gov.br/) ${ }^{17}$. 
Para a construção dos mapas temáticos, adotouse a malha municipal de Pernambuco, em formato shapefile em coordenadas geodésicas SIRGAS 2000, coletada no sítio eletrônico do IBGE ${ }^{14}$.

Para a análise de correlação entre as covariadas população (POPULAÇAO), área (ÁREA), densidade demográfica (DENSIDADE), índice de Gini (GINI), taxa de urbanização (TX_URB), IDH-M e percentual de estabelecimentos econômicos de toda natureza (COMERCIO) entre si, e entre elas e o coeficiente de incidência para RMR e interior, realizou-se o teste de Kendall ${ }^{18}$ (significância 5\%), utilizando-se linguagem R.

$\mathrm{O}$ coeficiente de incidência para cada município foi obtido através da fração entre os casos confirmados de Covid-19 no período do estudo (12/03 a 30/06/2020) e a população dos municípios estimada para 2019 (por 100.000 habitantes). Este foi suavizado pelo método bayesiano empírico local, devido às flutuações aleatórias dos coeficientes brutos ${ }^{16}$. Para visualizar a distribuição dos coeficientes (brutos e suavizados) no espaço, foram produzidos mapas temáticos utilizando-se intervalos de quartis. Em seguida, calculou-se o índice de Moran global, e realizou-se o teste de significância $(\mathrm{p}<0,05)$ a partir do coeficiente de incidência da média e da matriz de vizinhos ponderada por linha. Este índice mensura a correlação de uma variável com suas vizinhas no espaço, com valores variando entre -1 e +1 , sendo que índices positivos identificam autocorrelação direta, índices negativos, autocorrelação inversa e zero, aleatoriedade espacial ${ }^{19}$.

Visto que houve autocorrelação significativa, computou-se o Indicador Local de Associação Espacial (Local Indicator of Spatial Association - LISA) ${ }^{20}$, e efetuou-se o teste para verificar a existência de associação espacial significativa $(\mathrm{p}<0,05)$, utilizando-se o software GeoDa, versão 1.14. Objetivou-se, com isso, identificar agrupamentos espaciais significantes, os municípios de altas e baixas incidências e os de transição, admitindo-se a comparação do coeficiente de cada município com os valores dos vizinhos, para fins de constatação da dependência espacial, além da identificação de clusters espaciais (aglomerados) $)^{16,19}$.

Finalmente, os municípios foram classificados em quatro grupos: Q1, alto/alto (município acima da média com vizinhos acima da média); Q2, baixo/baixo (município abaixo da média com vizinhos abaixo da média); Q3, alto/baixo (município acima da média com vizinhos abaixo da média); e Q4, baixo/alto (municípios abaixo da média com vizinhos acima da média). Foram produzidos mapas a partir dos índices de Moran considerando-se os municípios com diferenças estatisticamente significantes $(\mathrm{p}<0,05)^{19}$.

Este trabalho não careceu de aprovação em Comitê de ética dado que se utiliza de dados de domínio público.

\section{Resultados}

A Figura 2 mostra a evolução espacial e temporal do número de casos de Covid-19 em Pernambuco, com distribuição semanal entre 19/03 e 23/04 (SE 12 a 17 - Figura 2A-F); após esse período, como o número de casos já era bastante significativo a ponto de não apresentar mudanças semanais aparentes, os dados foram representados quinzenalmente, integrando as SE 19, 21, 25 e 27 (Figura 2G-J).

$\mathrm{Na}$ primeira semana, em paralelo ao registro do caso índice (alóctone) em Recife em 12 de março (SE 12), despontam Belo Jardim (interior) e Jaboatão dos Guararapes (RMR) apresentando casos, sendo este último contíguo a Recife, ambos cortados pela BR-232, todos alóctones. Na SE 13 (Figura 2B) unem-se a eles Caruaru e Petrolina (interior), e Olinda, vizinho de Recife. $\mathrm{Na} 3^{\mathrm{a}}$ semana (SE 14), mais 6 municípios apresentaram casos, sendo 5 deles na região metropolitana, 4 circundantes a Recife. Na mesma semana, surgiram casos em municípios não vizinhos de Recife, mas cortados pela BR-101 (Figura 2C).

Pouco mais de um mês após o primeiro registro, a Figura 2E destaca Recife, Olinda e Jaboatão dos demais como aqueles com maior número de casos no estado. No interior, para essa mesma semana, o acumulado de casos corresponde a apenas $10 \%$ do estado, embora os municípios de Caruaru, Cachoeirinha e Vitória já registrassem números competitivos com a RMR. A partir da $6^{\circ}$ semana despontam Petrolina, Ipubi, Salgueiro, Arcoverde, Paudalho, Carpina, Amaraji e Pombos, todos no interior. Recife, Jaboatão e Olinda seguem com $82 \%$ dos casos da RMR.

Na SE 19, o arcabouço em torno de Recife está representado na Figura 2G. Atentou-se para o fato de que os municípios do interior, cortados pela BR-232, apresentaram mais casos que os seus vizinhos (Figuras $2 \mathrm{H}$ e $2 \mathrm{I}$ ). No dia 30/06, a conjectura da pandemia de Covid-19 reflete que apenas dois municípios (Mirandiba e Manari) não registraram casos.

A Tabela 1 mostra aspectos relacionados à morbidade por Covid-19, bem como ambientais, demográficos, sociais e econômicos das regiões 

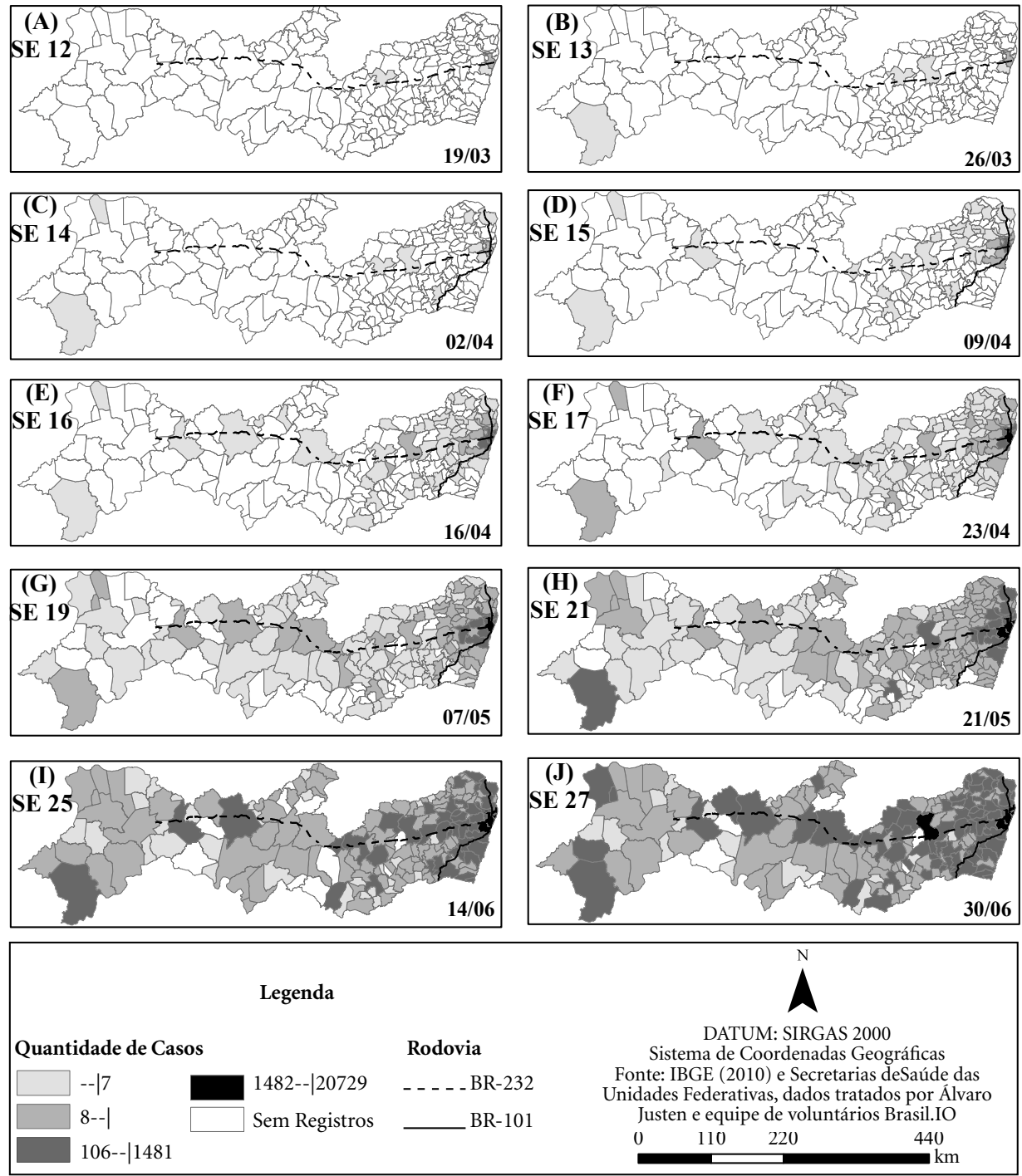

DATUM: SIRGAS 2000 Sistema de Coordenadas Geográficas Fonte: IBGE (2010) e Secretarias deSaúde das Unidades Federativas, dados tratados por Álvaro Justen e equipe de voluntários Brasil.IO \begin{tabular}{rrrr}
0 & 110 & 220 & 440 \\
\hline
\end{tabular}

Figura 2. Evolução espacial e temporal do número bruto de casos de covid-19 por SE, estratificado por intervalos geométricos. Pernambuco, 2020.

Fonte: autoria própria.

metropolitana e interior, e dos primeiros municípios a apresentarem casos alóctones (Recife e Jaboatão na RMR e Belo Jardim e Petrolina no interior) e autoctones (Olinda e Goiana na RMR e Palmares e Caruaru no interior) em cada região. Percebe-se que, até o fim do período de análise (30 de junho), 64\% dos casos se encontravam na RMR. Destaca-se que a RMR possui as cidades mais populosas: Recife, Jaboatão dos Guararapes e Olinda, que eram as que mais registraram ca- sos. No interior despontam Caruaru e Palmares com os maiores coeficientes, sendo Caruaru uma das cidades mais populosas.

A área territorial do interior é consideravelmente maior que a da RMR, e sua população corresponde a $57 \%$ da população do estado. Mesmo sendo maior, faz-se necessário considerar que esta grande área territorial deixa clara a disparidade de concentração de pessoas. Isso pode ser observado quando se compara densidade popu- 


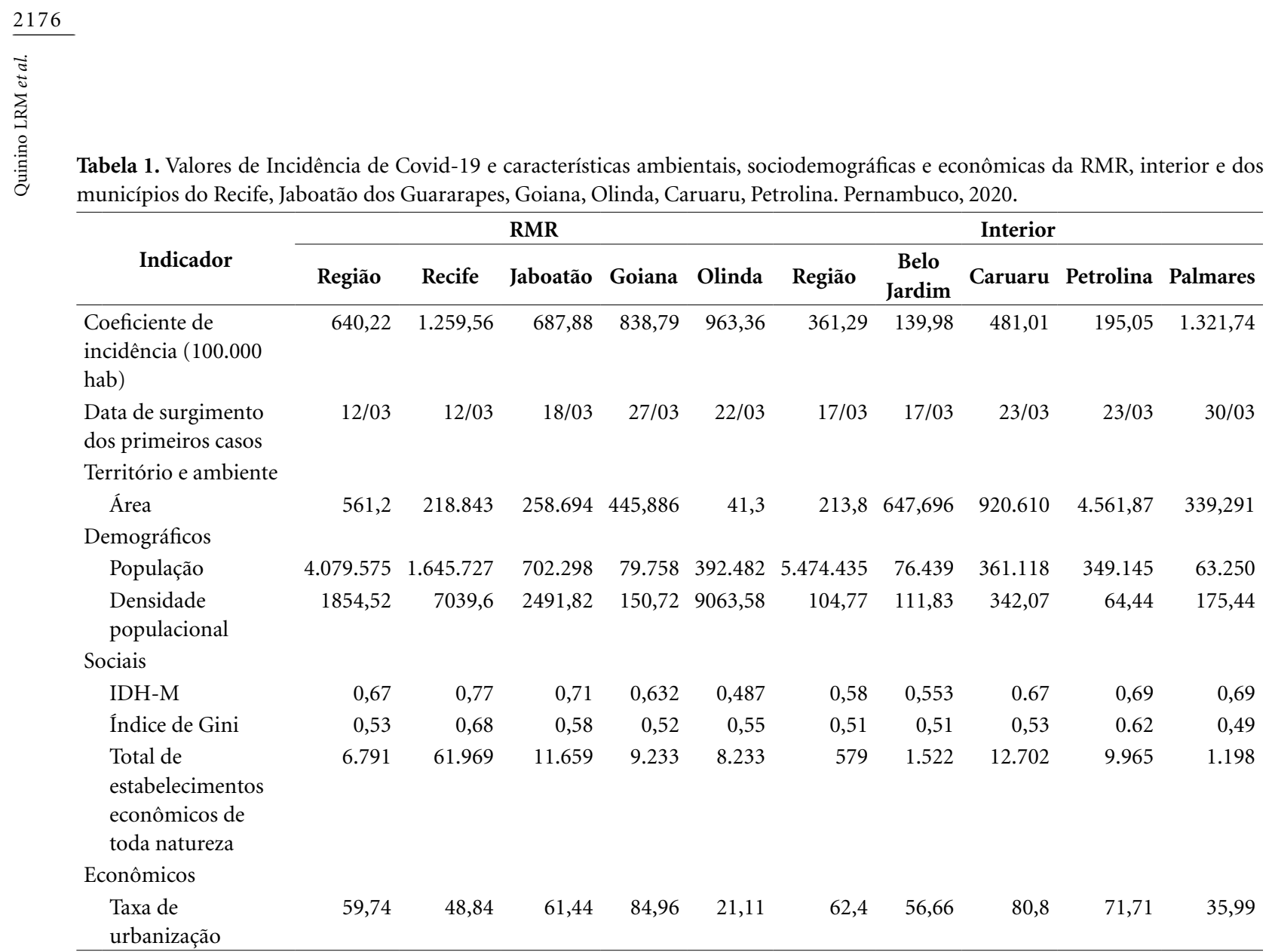

Fonte: Bases de dados Estaduais, Instituto Brasileiro de Geografia e Estatística (IBGE).

lacional, que é maior na RMR e nas três cidades a ela pertencentes (Tabela 1). Com relação às características sociais, percebe-se que o IDH-M é matematicamente maior na RMR e nos três municípios com maior densidade populacional, mas isso à custa de concentração de riquezas, evidente ao se observar o índice de Gini, ligeiramente maior nestes municípios e nesta região.

A Figura 3 (A e B) ilustra os valores significantes da correlação de Kendall entre as covariadas IDH-M, taxa de urbanização, Índice de Gini, densidade demográfica, área, população e percentual de estabelecimentos comerciais entre si, e entre estas e o coeficiente de incidência de Covid-19 para RMR e interior. Na RMR, observou-se correlação significativa positiva (significância 5\%) entre o coeficiente de incidência e a população e a densidade demográfica, e negativa entre taxa de urbanização e IDH-M, indicando que quanto maior a população e a densidade demográfica e menor a taxa de urbanização e o IDH-M, maior o número de casos. Já para o Interior, observou-se correlação significativa positiva entre o coeficiente de incidência e a população, densidade demográfica e taxa de urbanização, e negativa entre área. Foi possível, ainda, verificar que, mesmo não havendo correlação entre taxa de urbanização e a incidência no interior, a correlação positiva entre aquela e o IDH-M permitiu, indiretamente, inferir uma influência desta correlação na taxa de casos, isto é, quanto maior a taxa de urbanização maior o IDH-M, maior o coeficiente de incidência.

A Figura 4 mostra a comparação da distribuição espacial do coeficiente bruto de incidência por Covid-19 em Pernambuco (3A) com o coeficiente suavizado (3B) e o LISA com o coeficiente suavizado (3C). Verificou-se que a maioria dos municípios que possuem taxa de incidência acima de 506 casos por 100.000 hab estão na RMR, Zona da Mata e Agreste. A taxa suavizada (Figura $3 \mathrm{~B})$ evidenciou que os municípios com os $25 \%$ maiores coeficientes de incidência de Covid-19 (acima de 108/100.000 hab) estão distribuídos pela RMR, Zona da Mata pernambucana e, em menor quantidade, pelo Agreste e Sertão. 
(A)

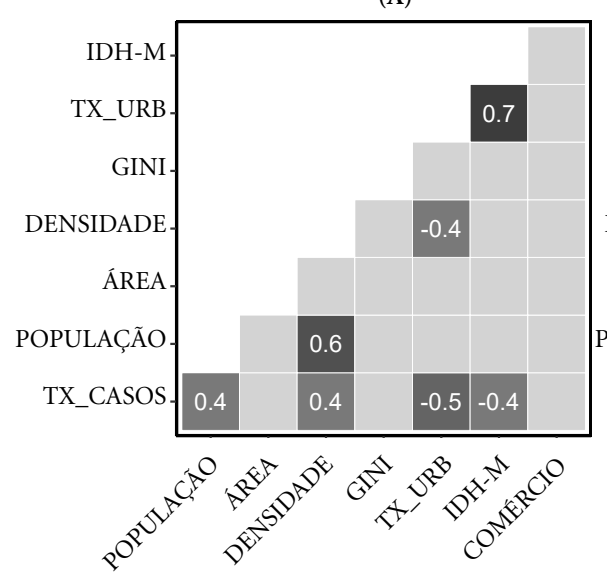

(B)
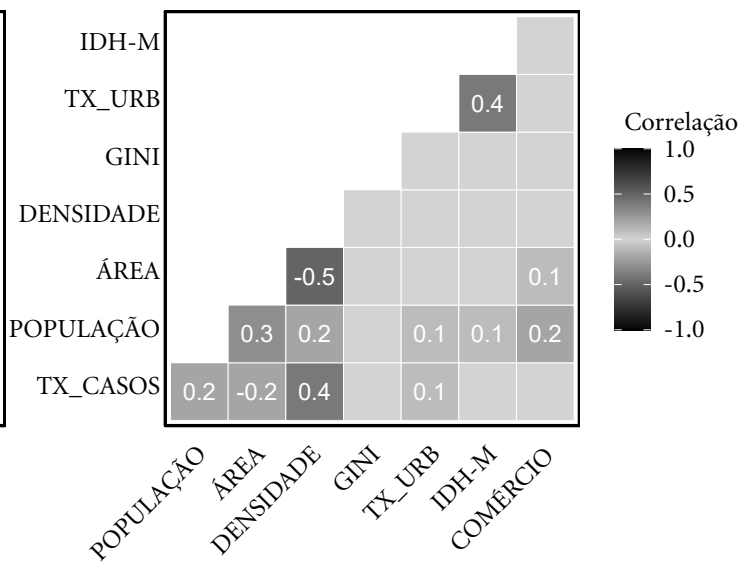

Figura 3. Matriz de correlação de Kendall da taxa de casos de covid-19 por (A) RMR e (B) Interior. Pernambuco, 2020.

Fonte: autoria própria.
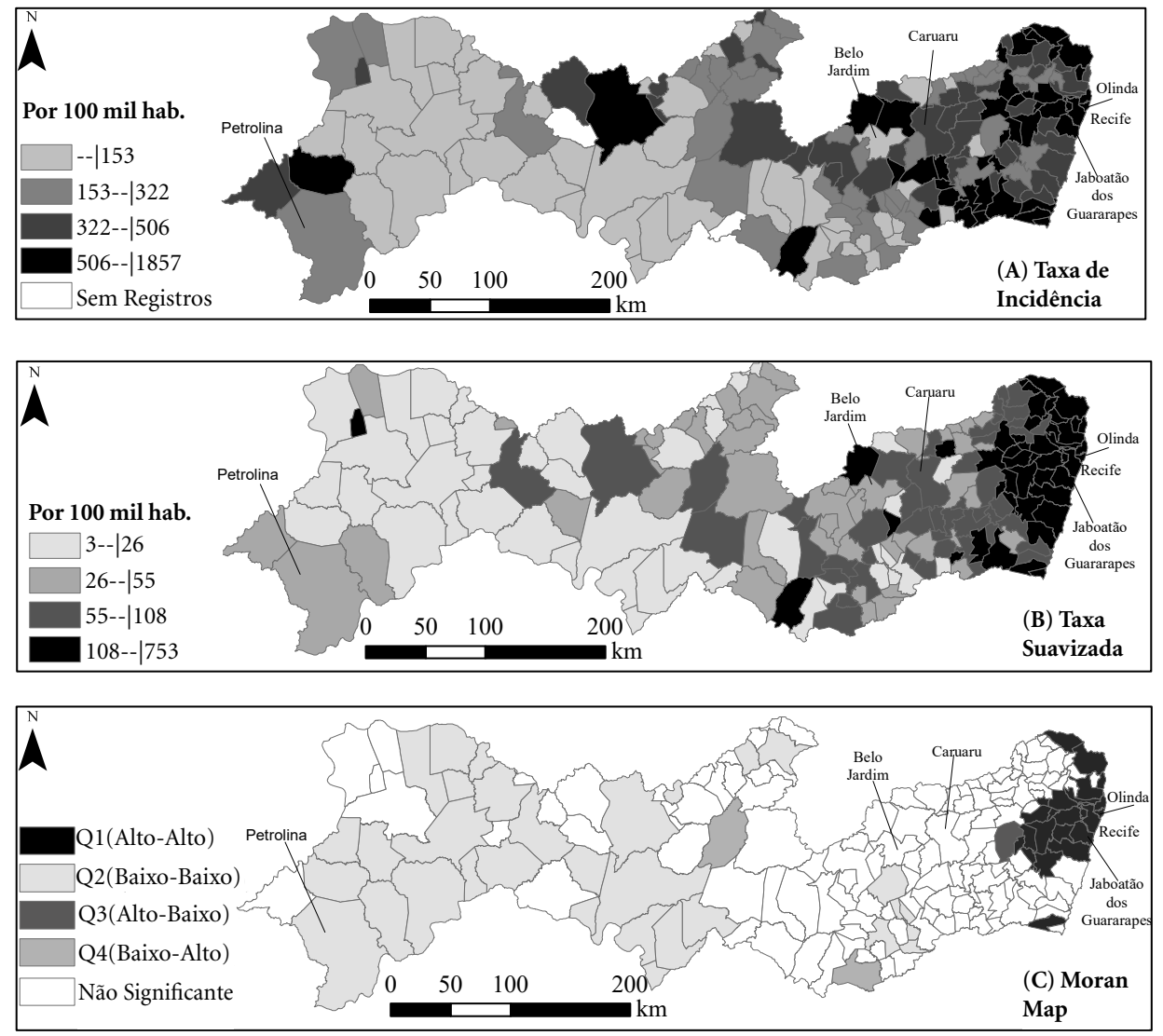

Figura 4. Coeficiente de incidência por Covid-19 (por 100.000 habitantes): coeficiente bruto por quartis (A), coeficiente suavizado por quartis (B) e LISA (Moran Map) (C), Pernambuco, 2020. 
A associação espacial obtida a partir do teste de Moran Global foi significativa, com o índice dos coeficientes suavizados igual a 0,679 $(\mathrm{p}<0,05)$, indicando que a taxa de incidência está correlacionada com o espaço. Os clusters de municípios com os maiores coeficientes de incidência com vizinhos com altas taxas (Q1) concentraram-se na RMR, enquanto que aqueles com baixos coeficientes de incidência com vizinhos baixos (Q2), encontram-se nas mesorregiões do Sertão e São Francisco.

\section{Discussão}

No momento da redação deste manuscrito, embora a expansão da Covid-19 para áreas rurais estivesse sendo cogitada e abordada em trabalhos no Brasil ${ }^{7,21}$ e no mundo ${ }^{22,23}$, nenhum estudo foi detectado abordando este fenômeno na Região Nordeste do Brasil, especificamente em Pernambuco. Adentrando nas conclusões deste trabalho e considerando o período do estudo, a pandemia Covid 19 pareceu ter um efeito desproporcional nas regiôes metropolitana e interior (rural) de Pernambuco, mostrando concentração de casos nas áreas urbanas.

A evolução espacial e temporal mostrou que os primeiros casos conhecidos de Covid-19 ocorreram na RMR: Recife (12/03/2020 - SE 12), Jaboatão dos Guararapes (17/03/2020 - SE 12) e Olinda (23/03/2020 - SE 13), sendo os casos de Recife e Jaboatão, importados e o de Olinda, autóctone ${ }^{24}$. Os municípios do interior que inicialmente apresentaram casos autóctones foram, principalmente, os que margeiam as $\mathrm{BR}$ 101 e 232. Belo Jardim (17/03/2020 - SE 12) surge como a primeira a notificar casos, depois do que emergem Caruaru (24/03/2020) e Petrolina (19/03/2020), todos alóctones. Em seguida, vem Palmares (30/03/2020) com possível transmissão Autóctone ${ }^{25}$.

A caracterização das regiões e dos municípios mostrou que a RMR e seus municípios, mesmo tendo menor área, população geral e taxa de urbanização, possuem as maiores densidades demográficas, maior concentração de estabelecimentos econômicos e, apesar de possuírem os maiores IDH, possuem os maiores índices de Gini.

A análise de correlação para a RMR mostrou que quanto maior a população e a densidade demográfica e quanto menor o IDH-M e a taxa de urbanização, maior o número de casos. A análise para o interior mostrou que quanto mais popu- loso, com maiores densidade populacional e taxa de urbanização e quanto menor a área, maior o número de casos. Os mapas mostraram que a região com maior risco de ocorrência para Covid-19 foi a Metropolitana.

Estudos realizados em âmbito nacional ${ }^{7} \mathrm{e}$ internacional ${ }^{23,26}$ têm ressaltado a importância de se compreenderem as particularidades destas populações na expansão da Covid-19. De um lado, têm-se as regiões metropolitanas, cujas características gerais incluem alto tráfego aéreo e marítimo internacional, alta densidade demográfica e mobilidade urbana, fatores conhecidos por facilitar a propagação das doenças ${ }^{27}$.

Do outro, tem-se as áreas do interior, rurais e/ ou remotas, conhecidas por ter baixa densidade demográfica na escala municipal e pela dificuldade de acesso a bens e serviços complexos $(\mathrm{IBGE})^{14}$. A priori, estas características parecem atuar reduzindo a velocidade de transmissão da Covid-19, porém, estas áreas costumam abrigar populações em situação de vulnerabilidade e extrema pobreza, difíceis de serem alcançadas por políticas públicas, o que pode atuar agravando o quadro nestas regiões ${ }^{7,23,26}$.

Considerando estas diferenças, neste momento, entender como se deu a disseminação dos casos de Covid-19 em Pernambuco se faz necessário. $\mathrm{O}$ padrão de transmissão local que se deu em Pernambuco pareceu ser decorrente da introdução da doença em cidades cujo crescimento populacional não foi acompanhado de desenvolvimento urbano ${ }^{28-30}$, conforme apoiado pelos resultados da análise de correlação. Este fato segue a tendência mundial de que, embora o Sars-Cov-2 tenha adentrado os países pelas camadas sociais mais abastadas, sua expansão se dá em populações economicamente desfavorecidas e penaliza mais aqueles locais com maiores desigualdades sociais, altas densidades populacionais, e baixos IDH e taxas de urbanizaçãa ${ }^{28-33}$.

Dois conceitos intrinsecamente ligados que permitem entender a transmissão local da Covid-19 são a rede de mobilidade intercidades e a mobilidade pendular. O primeiro atua balizando a rede de transmissão, condição necessária para o surgimento de surtos de doenças, por permitir que a mesma seja levada de um lugar a outro ${ }^{34}$. Já a mobilidade pendular (MP) é caracterizada por movimentos cotidianos das pessoas entre o local de residência e locais de trabalho ou estudo ${ }^{35}$.

A importância da migração pendular na disseminação de doenças foi abordada por estudo realizado na região de tríplice fronteira Brasil-Argentina-Paraguai, em 2017. Os autores 
apontaram que nas áreas que possuem maior e mais diversificado mercado de trabalho e oferta de instituições de ensino é onde ocorre a maior mobilidade pendular e, portanto, onde há maior risco de transmissão de doenças. Este fato, somado às precárias estruturas sociais ${ }^{36,37}$, favorece a disseminação de doenças no geral, o que pareceu propiciar a disseminação da Covid-19 em Olinda (autoctonia) ${ }^{38}$ e arredores, e em Camaragibe, São Lourenço da Mata e Goiana.

No caso de Pernambuco, ressaltam-se as intrínsecas relações existentes entre Olinda e a capital Recife. Olinda é conhecida como cidade dormitório: pessoas empregadas em Recife optam por viver em Olinda porque pagam por habitações mais baratas, favorecendo este arranjo pendular ${ }^{39}$. Desta forma, pode-se supor que a contaminação tenha se dado em Recife que, por sua vez, apresenta um histórico de ocupações inapropriadas e profundo adensamento. Sobre a expansão pra Goiana, localizada na Mata Norte, pode-se mencionar, a existência de grandes empreendimentos, como um polo farmacoquímico e um polo automotivo ${ }^{40}$, que favorece alto tráfego de pessoas. Além disso, esta cidade é cortada pela BR $101^{35,40}$.

Com relação à rede de mobilidade intercidades, estudos realizados na Coreia do Sul ${ }^{41}$ e em Hong $\mathrm{Kong}^{42}$. respaldam as conclusões abaixo descrita quando esclarecem a relação entre o volume de tráfego e a disseminação da Covid-19.

No interior, depois de Belo Jardim, Caruaru e Petrolina, tem-se Palmares como a próxima a apresentar casos, já autóctones ${ }^{38}$. Palmares é servida pelas BR 101 sul, além das rodovias PE 96 (litoral), PE 103 (interliga Palmares à rodovia 232), PE 120 (que vai até Caruaru) e PE 126 (que liga a cidade à Garanhuns). Além disso, ela dista apenas $75 \mathrm{~km}$ do Complexo Industrial Portuário Governador Eraldo Gueiros, ou porto de Suape, que é o maior porto público da região Nordeste apresentando grande fluxo de pessoas ${ }^{43}$.

Outra BR que cruza importantes municípios sede de polos de desenvolvimento de Pernambuco, a exemplo de Caruaru, e que pode ter funcionado como agente propulsor da disseminação da Covid-19 no interior de Pernambuco é a 232, interligando RMR, Agreste e Sertão. Caruaru é a maior cidade do Agreste, sendo rodeada por Santa Cruz do Capibaribe e Toritama, que apresentam alto vigor econômico, o que favorece o fluxo de pessoas $^{44}$.

A análise da autocorrelação espacial dos coeficientes suavizados possibilitou a identificação de clusters de risco de ocorrência de Covid-19, neutralizando-se a influência de valores extremos e discrepantes. Este ajuste proporciona maior estabilidade no indicador, com base nos coeficientes dos setores vizinhos que compõem a área de estudo ${ }^{19}$. A divisão de classes por intervalos geométricos foi escolhida por ser a que melhor revelou a complexidade na distribuição do coeficiente de incidência da Covid-19, permitindo uma divisão mais equitativa. A utilidade deste tipo de análise para determinação de áreas de risco de transmissão para Covid-19 pode ser vista em trabalho realizado no Ceará em $2020^{45}$.

Viu-se que as regiões Q1 estão localizadas na RMR e em seus arredores (Zona da Mata Pernambucana), municípios mais populosos, porém com baixas taxas de urbanização e IDHM. Observou-se, também, que as áreas Q2 tomam a maioria do sertão. Existe apenas uma área Q3 no Agreste pernambucano e duas áreas Q4, sendo uma no Agreste e outra no Sertão, mostrando tendência de interiorização da Covid-19 em Pernambuco.

Arrematando os achados acima, ao se considerar a análise de correlação realizada neste estudo viu-se que, tanto para a RMR quanto para o interior, fatores como alta densidade populacional e más condições de vida em geral estiveram relacionados com altas incidências da Covid-19. Assim, mesmo que os casos estejam concentrados na RMR cabe tanto considerar estes fatores como importantes na manutenção de altas incidências na RMR quanto na perpetuação da Covid-19 para o interior, o que se reveste de maior gravidade sabendo que esta é uma população historicamente carente e com dificuldades de acesso a serviços de saúde. Além disso, o aumento dos casos de Covid-19 no interior pode colapsar os serviços de saúde pelo aumento geral da deman$\mathrm{da}^{6-10,11,21-23}$.

Estes desfechos e suas explicações são apoiados por estudos. Um trabalho determinou associações da Covid-19 com raça, saúde e desigualdade econômica nos Estados Unidos, mostrando que residentes em locais densamente povoados e com menores níveis de renda correm maior risco de infecção por Covid-1929. Outro mostrou que a alta concentração de pessoas teve contribuição no aumento do número de casos de Covid-1933. Já a expansão preferencial da Covid-19 em zonas urbanas, vista neste estudo, é reforçada por outros trabalhos $5,6,9,10,21,27,30,33,45$.

Estas observações são importantes tanto para a pesquisa epidemiológica quanto para os serviços de saúde. No tocante à epidemiologia, cita-se o fato de ser possível visualizar precisamente as 
áreas de maior risco de contaminação para Covid-19. Quanto aos serviços de saúde, é possível planejar a resposta política de maneira mais acertada, dada a existência visível e comprovada de áreas mais carentes de ações específicas. Pode-se, ainda, levar em conta as diferenças matemáticas entre os indicadores de território, sociodemográficas e econômicas entre o interior e a RMR no planejamento das ações de saúde, sendo estes fatores que devem ser considerados na expansão e na manutenção do ciclo de transmissão da Covid-19 em Pernambuco.

Como limitações, inicia-se mencionando que o período do estudo foi entre 12/03 e 30/06, quando a pandemia foi relatada predominantemente nas áreas metropolitanas. No entanto, na última parte do período de estudo, já se observavam casos em todo o estado, com presença de áreas de transição, o que pode implicar que a pandemia está gradualmente se instalando nas áreas rurais. Estudos subsequentes, usando dados por um período maior de tempo, são recomendados para melhores conclusões. Também existe o fato de que os dados utilizados, embora provenientes de sistemas oficiais, são secundários, sendo passíveis de viés de informação ao se levar em conta o caminho percorrido desde a ocorrência do evento até a inserção dos dados nos sistemas. Outra limitação está associada ao sub-registro de dados pelos serviços locais de saúde, refletido na redução do número de casos.

\section{Colaboradores}

LRM Quinino, IS Diniz, FHM Vasconcellos e YTM Ramos participaram da concepção, planejamento, análise, interpretação e redação do manuscrito. LR Aguiar e F De Bastiani participaram da análise, interpretação, redação e revisão final do manuscrito.
Por último, sabe-se que o Brasil é um dos países com menor número de testes por habitante, o que se agrava em áreas rurais, dado que estas têm menos disponibilidade em comparação com os residentes as áreas metropolitanas. Isso pode levar a pandemia a progredir silenciosamente, sem detecção e, eventualmente, evoluir para situações graves e colapso do sistema de saúde.

$\mathrm{O}$ conhecimento epidemiológico utilizado neste artigo para entender a interiorização da Covid-19 em Pernambuco é de extrema importância para o campo da saúde coletiva, no momento em que elucida os fatores relacionados a este evento visando melhor eficácia das ações de enfrentamento. Mesmo que a disseminação da Covid-19 siga um padrão mundial, com entrada e contaminação inicial em regiões metropolitanas e espalhamento para zonas rurais, a aplicabilidade das conclusões deste estudo deve ser vista com cautela, dadas as diferenças econômicas e sociais entre os países. As principais áreas de risco para Covid-19 em Pernambuco situam-se na RMR, área mais desenvolvida, porém com más condições sociais. Sugere-se a realização de estudos para estimar a associação entre variáveis sociodemográficas, econômicas e ambientais e a ocorrência de Covid-19 na RMR e no interior, a fim de determinar estatisticamente as diferenças existentes. Adicionalmente, recomenda-se o direcionamento de esforços para reduzir a transmissão da Covid-19 na RMR e Zona da Mata pernambucana. 


\section{Referências}

1. Zhu N, Zhang D, Wang W, Li X, Yang B, Song J, Xiang Z, Baoying H, Weifeng S, Roujian L, Peihua N, Faxian Z, Xuejun M, Dayan W, Wenbo X, Guizhen W, George FG, Phil D, Wenjie T. A Novel Coronavirus from Patients with Pneumonia in China, 2019. J Engl J Med 2020; 382(8):727-733.

2. World Health Organization (WHO). International health regulations (2005) [Internet]. $3^{\text {a }}$ ed. 2016 [acesssado 2020 jun 20]. Disponível em: https:// apps.who.int/iris/bitstream/handle/10665/246107/ 9789241580496-eng.pdf; jsessionid=1F43A65C3AC917BD8FAABB0FB2E88565? sequence $=1$.

3. Van Bavel JJ, Baicker K, Boggio P, Capraro V, Cichocka A, Cikara M, Crockett M, Crum AJ, Douglas KM, Druckman JN, Drury J, Dube O, Ellemers N, Finkel EJ, Fowler JH, Gelfand M, Han S, Haslam SA, Jetten J, Kitayama S, Mobbs D, Napper LE, Packer DJ, Pennycook G, Peters E, Petty RE, Rand DG, Reicher SD, Schnall S, Xarife A, Skitka LJ, Smith SS, Sunstein CR, Tabri N, Tucker JA, Linden SVD, Lange PV, Weeden KA, Wohl MJA, Zaki J, Zion SR, Willer R. Using social and behavioural science to support COVID-19 pandemic response. Nat Human Behav 2020; 4:460-471.

4. Chung RY, Dong D, Li MM. Socioeconomic gradient in health and the covid-19 outbreak. BMJ 2020; 369.

5. Peters DJ. Community Susceptibility and Resiliency to COVID-19 Across the Rural-Urban Continuum in the United States. J Rural Health 2020; 36(3):446-456.

6. Chen X, Chen H. Differences in Preventive Behaviors of COVID-19 between Urban and Rural Residents: Lessons Learned from A Cross-Sectional Study in China. Int J Environ Res Public Health 2020; 17(12):e4437.

7. Mayara F, Mendes FC, Cecilia M, Vieira SK, Reis CB, Lima SVX, Werreria NS, Duarte DR. A pandemia de COVID-19 em territórios rurais e remotos: perspectiva de médicas e médicos de família e comunidade sobre a atenção primária à saúde. Cad Saude Publica 2020; 36(7):e00108920.

8. Cabral SAPS, Souza MLT, Vieira SW. Síndrome Respiratória Aguda Grave em Pernambuco: comparativo dos padrões antes e durante a pandemia de COVID-19. Cien Saude Colet 2020; 25(Supl. 2):41414150.

9. Jesus JG, Sacchi C, Candido DS, Claro IM, Sales FCS, Manuli ER, Silva DBB, Paiva TM, Pinho MAB, Santos KCO, Hill SC, Aguiar RS, Romero F, Santos FCP, Gonçalves CR, Timenetsky MC, Quick J, Croda JHR, Oliveira W, Rambaut A, Pybus OG, Loman NJ, Sabino EC, Faria NR. Importation and early local transmission of COVID-19 in Brazil, 2020. Rev Inst Med Trop 2020; 62(30):1-5.

10. Silva B. A interiorização da Covid-19 no Nordeste: temos infraestrutura de saúde? [Internet]. Conselho Regional de Economia (CORECON); 2020 [acessado 2020 jul 24]. Disponível em: http://www.corecon-rn. org.br/2020/04/07/a-interiorizacao-da-covid-19-nonordeste-temos-infraestrutura-de-saude/.

11. Marinelli NP, Albuquerque LPA, Sousa IDB, Batista FMA, Mascarenhas MDM, Rodrigues MTP. Evolução de indicadores e capacidade de atendimento no início da epidemia de COVID-19 no Nordeste do Brasil, 2020. Epidemiol Serv Saude 2020; 29(3):e2020226.
12. Condepe Fidem. Recife: Plano de Desenvolvimento Urbano Integrado [Internet]. 2020 [acessado 2020 jul 08]. Disponível em: https://pdui-rmr.pe.gov.br/ oPDUI.

13. Secretaria de Saúde de Pernambuco. Plano Estadual de Saúde 2020-2023. Pernambuco [Internet]. 2020 [acessado 2020 jun 29]. Disponível em: http://portal. saude.pe.gov.br/sites/portal.saude.pe.gov.br/files/plano_estadual_de_saude_2020-2023.pdf.

14. Brasil. Instituto de Geografia e Estatística (IBGE) [Internet]. [acessado 2020 jul 01]. Disponível em: https:// cidades.ibge.gov.br/brasil/pe/recife/panorama.

15. Brasil. Ministério da Saúde (MS). Painel Coronavírus [Internet]. [acessado 2020 jul 01]. Disponível em: https://covid.saude.gov.br/.

16. Marshall RJ. Mapping disease and mortality rates using empirical bayes estimators. J Roy Stat Soc C Appl Stat 1991; 40(2):283-294.

17. Pernambuco. Banco de Dados do Estado [Internet]. [acessado 2020 jul 01]. Disponível em: http://www. bde.pe.gov.br/estruturacaogeral/conteudo_site2.aspx.

18. Hollander M, Wolfe DA, Chicken E. Nonparametric statistical methods. $3^{\text {a }}$ ed. Nova Jersey: John Wiley \& Sons; 2014.

19. Brasil. Ministério da Saúde (MS). Fundação Oswaldo Cruz (Fiocruz). Introdução à estatística espacial para a saúde pública [Internet]. 2007 [acessado 2020 jun 29]. Disponível em: http://www.escoladesaude.pr.gov. br/arquivos/File/TEXTOS_CURSO_VIGILANCIA/ capacitacao_e_atualizacao_em_geoprocessamento_ em_saude_3.pdf.

20. Anselin L. Local indicators of spatial association Lisa. Geographical analysis. Wiley Online Library 1995; 27(2):93-115.

21. Fundação Oswaldo Cruz (Fiocruz). Monitora Covid-19. Tendências atuais da pandemia de Covid-19: Interiorização e aceleração da transmissão em alguns estados. Nota Técnica [Internet]. 2020 [acessado 2020 jun 29]. Disponível em: https://revistafitos.far.fiocruz. $\mathrm{br} /$ fitos/index.php/noticias/tendencias-atuais-da -pandemia-de-covid-19-interiorizacao-e-aceleracaoda-transmissao-em-alguns-estados.

22. Dandachi D, Reece R, Wang EW, Nelson T, Rojas-Moreno C, Shoemaker M. Treating COVID-19 in Rural America. J Rural Health 2021; 37:205-206.

23. Henning-Smith C, Tuttle M, Kozhimannil KB. Unequal Distribution of COVID-19 Risk Among Rural Residents by Race and Ethnicity. J Rural Health 2021; 37:224-226.

24. Secretaria de Saúde de Pernambuco. Pernambuco confirma 2 casos importados de covid-19 [Internet]. 2020 [acessado 2020 jun 28]. Disponível em: https://drive. google.com/file/d/1Q05y4zyE1eYgbn5ScCk4CPZ_ D5i5qbX2/view.

25. Secretaria de Saúde de Pernambuco. Boletim covid-19 SES-PE [Internet]. 2020 [acessado 2020 jun 28]. Disponível em: https://drive.google.com/file/d/1YF0jDnWyu0qtZ2ONNVyvegHulPZeRKxh/view.

26. Karim SA, Chen H-F. Deaths from COVID-19 in Rural, Micropolitan, and Metropolitan Areas: A County-Level Comparison. J Rural Health 2021; 37(1):124132. 
27. Yip TL, Huang Y, Liang C. Built environment and the metropolitan pandemic: Analysis of the COVID-19 spread in Hong Kong. Build Environ 2021; 188:107471.

28. Nery MB, Souza AAL, Adorno S. Os padrões urbano-demográficos da capital paulista. Estud Av 2019; 97(33):5-36

29. Abedi V, Olulana O, Avula V, Chaudhary D, Khan A, Shajouei S, Li J, Zand R. Racial, Economic and Health Inequality and COVID-19 Infection in the United States. J Racial Ethn Health Disparities 2020; 1-11.

30. Ramírez IJ, Lee J. COVID-19 Emergence and Social and Health Determinants in Colorado: A Rapid Spatial Analysis. Int J Environ Res Public Health 2020; 17(11):3856

31. Olulana O, Abedi V, Avula V, Chaundhary D, Khan A, Shanjouei S, Li J, Zand R. Regional Association of Disability and SARS-CoV-2 Infection in 369 Counties of the United States. medRxiv 2020 [preprint].

32. Niedzwiedz CL, O’Donnell CA, Jani BD, Demou E, Ho FK, Celis- Morales C, Nicholl BI, Mair FS, Welsh P, Sattar N, Pell JP, Katikireddi SV. Ethnic and socioeconomic differences in SARS-CoV-2 infection: prospective cohort study using UK Biobank. BMC Med 2020; 18(1):160.

33. Roy S, Khalse M. Epidemiological Determinants of COVID-19-Related Patient Outcomes in Different Countries and Plan of Action: A Retrospective Analysis. Cureus 2020; 12(6):e8440.

34. Freitas VLS, Konstantyner TCRO, Mendes JF, Sepetauskas CSN, Santos LBL. The correspondence between the structure of the terrestrial mobility network and the emergence of COVID-19 in Brazil. Cad Saude Publica 2020: 36(9):e00184820.

35. Zaslavsky R, Goulart BNG. Migração pendular e atenção à saúde na região de fronteira. Cien Saude Colet 2017; 22(12):3981-3986.

36. Assis IS, Berra TZ, Alves LS, Ramos ACV, Arroyo LH, Santos DT, Arcoverde MAM, Alves JD, Crispim JA, Pieri FM, Frade MAC, Pinto IC, Nunes C, Arcêncio RA. Leprosy in urban space, areas of risk for disability and worsening of this health condition in Foz Do Iguaçu, the border region between Brazil, Paraguay and Argentina. BMC Public Health 2020; 20(1):119.

37. Ahmed S, Dávila JD, Allen A, Haklay MM, Tacoli C, Fèvre EM. Does urbanization make emergence of zoonosis more likely? Evidence, myths and gaps. Environ Urban 2019; 31(2):443-460.
38. Secretaria de Saúde de Pernambuco. Boletim Covid-19 SES-PE. Covid-19: Governador Paulo Câmara reforça rede de saúde com mais de 4,7 mil profissionais [Internet]. [acessado 2020 jun 28]. Disponível em: https:// drive.google.com/file/d/1Vqmk1G0vFWNRqWJe6zi03T1etukxZKhQ/view

39. Silva CCG, Mello SCB. Recife, Veneza Brasileira: repensando a mobilidade urbana a partir de seus rios. Cidad Comun Territ 2017; 34:110-132.

40. Lyra TM, Bezerra ACV, Albuquerque MSV. Os desafios dos Polos de Desenvolvimento na perspectiva dos atores sociais locais de Goiana, Pernambuco. Physis 2015; 4(25):1117-1139.

41. Lee H, Park SJ, Lee GR, Kim JE, Lee JH, Jung Y, Nam EW. The relationship between trends in COVID-19 prevalence and traffic levels in South Korea. J Infect Dis 2020; 96:399-407.

42. Yip TL, Huang Y, Liang C. Built environment and the metropolitan pandemic: Analysis of the COVID-19 spread in Hong Kong. Build Environ 2021; 188:107471.

43. Oliveira RS, Morais HMM, Goes PSA. Problematizando a díade saúde e desenvolvimento: o caso do polo de Suape no estado de Pernambuco. Saude Soc 2018; 1(27):149-162.

44. Matos FRN, Bastos AT, Machado DQ. Desenvolvimento local no Agreste pernambucano: uma "utopia possível”. Interações (Campo Grande) 2014; 1(15):147157.

45. Pedrosa NL, Albuquerque NLS. Spatial Analysis of COVID-19 cases and intensive care beds in the State of Ceará, Brazil. Cien Saude Colet 2020; 25(Supl. 1):2461-2468.

Artigo apresentado em 24/09/2020

Aprovado em 05/03/2021

Versão final apresentada em 07/03/2021

Editores-chefes: Romeu Gomes, Antônio Augusto Moura da Silva 